Article

\title{
High Performance Near-Infrared Emitters with Methylated Triphenylamine and Thiadiazolo[3,4-g]quinoxaline- Based Fluorophores
}

\author{
Youming Zhang, Chengjun Wu, Minrong Zhu and Jingsheng Miao *
}

check for updates

Citation: Zhang, Y.; Wu, C.; Zhu, M.; Miao, J. High Performance

Near-Infrared Emitters with

Methylated Triphenylamine and

Thiadiazolo[3,4-g]quinoxaline-Based Fluorophores. Molecules 2021, 26, 6386. https://doi.org/10.3390/ molecules 26216386

Academic Editor: Saulius

Grigalevicius

Received: 30 August 2021

Accepted: 20 October 2021

Published: 22 October 2021

Publisher's Note: MDPI stays neutral with regard to jurisdictional claims in published maps and institutional affiliations.

Copyright: (c) 2021 by the authors. Licensee MDPI, Basel, Switzerland. This article is an open access article distributed under the terms and conditions of the Creative Commons Attribution (CC BY) license (https:// creativecommons.org/licenses/by/ $4.0 /)$.
Shenzhen Key Laboratory of Polymer Science and Technology, College of Materials Science and Engineering, Shenzhen University, Shenzhen 518060, China; zhangym@szu.edu.cn (Y.Z.); 2070343051@email.szu.edu.cn (C.W.); zhumr@szu.edu.cn (M.Z.)

* Correspondence: jingshengmiao@szu.edu.cn

Abstract: Three near-infrared emitters (2TPA-QBT, 2MeTPA-BT and TPA-QBT-MeTPA) were rationally designed and synthesized. Density functional theory (DFT) and time-dependent density functional theory (TDDFT) calculations showed that the introduction of mono- or di-methyl groups between the donors and acceptor could result in the spatial configuration changing greatly for 2MeTPA-QBT and TPA-QBT-MeTPA compared to their parent compound 2TPA-QBT. The emission of TPA-QBT-MeTPA had a more obvious hybridized local and charge transfer feature (HLCT) based on the influence of the steric hindrance of the methyl substituent. Attributed to their different spatial configurations and luminescence mechanisms, different emission wavelengths with photoluminescent quantum yields of $26 \%, 38 \%$ and $34 \%$ in toluene, as well as $24 \%, 27 \%$ and $31 \%$ in 4,4'-bis(N-carbazolyl)-1,1'-biphenyl (CBP) doped film, were observed for 2TPA-QBT, 2MeTPA-QBT and TPA-QBT-MeTPA, respectively. The constructed organic light-emitting devices (OLEDs) displayed electroluminescence with emission peaks at 728,693 and $710 \mathrm{~nm}$, with maximum external quantum efficiencies of $1.58 \%, 1.33 \%$ and $3.02 \%$ for the 2TPA-QBT, 2MeTPA-QBT and TPA-QBTMeTPA-doped OLEDs, respectively. This work illustrated the effect of spatial configuration changes on the luminescence properties of donor-acceptor-type organic emitters.

Keywords: near-infrared emitters; hybridized local and charge transfer; organic light-emitting diodes; electroluminescence

\section{Introduction}

The tuning of the emission wavelength of organic light-emitting diodes (OLEDs) to the deep-red/near-infrared (DR/NIR) region has attracted widespread attention since DR/NIR light has a wide range of applications in night-vision displays, sensors and optical communications, as well as offering superior biocompatibility for medical systems [1-5]. To achieve this, tremendous efforts are devoted to achieving DR/NIR emission in the range of 650-900 nm from transition-metal complexes, boron dipyrromethene dyes and fluorescent materials with donor-acceptor (D-A) structures [5-8]. According to spin quantum theory, the ratio of the singlet to triplet exciton is 1:3 for exciton recombination process. Therefore, the utilization of the triplet exciton energy is the essential factor to obtain highly efficient devices [9-11]. Based on this, transition-metal complexes have attracted significant attention because they can provide a strong spin-orbit coupling between the triplet state and ground state to achieve $100 \%$ internal quantum efficiency [12-16]. Such devices based on transition-metal complexes can usually achieve high luminescence efficiency [17-19]. However, the sensitivity to oxygen and moisture, as well as the environmental toxicity and high costs, could limit the application and development of metal complexes-based OLEDs. Because of this, metal-free organic materials have attracted considerable research interest due to the advantages of their short exciton lifetime, tunable band gaps, molecular structure diversity, low costs, as well as their capability for batch production [20-23]. 
In the last decade, for utilizing both singlet and triplet excitons, hybridized local and charge-transfer state (HLCT) and thermally activated delayed fluorescence (TADF) materials was proposed by controlling the excited state and regulating the orbital separation between the highest occupied molecular orbital (HOMO) and the lowest unoccupied molecular orbital (LUMO), and break the 5\% external quantum efficiency (EQE) limit in OLEDs [20,22,24-28]. For HLCT emission, the reverse intersystem crossing (RISC) of excitons from the triplet state $\left(T_{n}\right)$ to singlet state $\left(S_{n}\right)$ with close energy levels can also occur in the excited state. This state combines both local excitation (LE) and charge transfer (CT) states into a special one that possesses two combined and compatible characteristics: a large transition moment from the LE state (cold exciton) and a weakly bound exciton from the CT state ("hot" exciton). For TADF, a smaller value of the $S_{1}-T_{1}$ band gap $\left(\Delta E_{\mathrm{ST}}\right)$ is beneficial for harvesting triplet excitons via reverse intersystem crossing (RISC). The value of $\Delta E_{\mathrm{ST}}$ is related to the overlap integral of the HOMO and the LUMO. DR/NIR emission materials based HLCT/TADF characteristics were also reported. For example, Liu et al. reported a D- $\pi$-A- $\pi$-D-type NIR emitter of NZ2TPA, which showed HLCT features and an EQE of 3.9\% with an emission peak at $696 \mathrm{~nm}$ in undoped electroluminescent device [29]. Li et al. reported a D-A-type DR/NIR emitter of TPA-QCN, with TADF properties and high EQEs of 3.9-14.5\% when doped under different concentrations in OLED devices [30].

In a previous study, we synthesized emitters with HLCT emission features by introducing methyl groups into small symmetric molecules [31]. The results showed that the introduction of a single methyl group (2MeTPA-BT) made the HLCT properties more obvious. Herein, in order to obtain NIR electroluminescent materials, a [1,2,5]thiadiazolo[3,4$g$ ]quinoxaline acceptor, which had a stronger electron-withdrawing ability and larger conjugation, was selected to replace benzo[c] [1,2,5]thiadiazole. A D-A-D/D'-type emitter of 2MeTPA-QBT was synthesized by introducing a single methyl group into each $\mathrm{D}$ unit. In addition, an asymmetric emitter, TPA-QBT-MeTPA, was obtained by introducing single methyl group into one of the D units. Their parent compound 2TPA-QBT was also synthesized for comparison (Figure 1). Due to steric hindrance, the torsional angles between the $\mathrm{D}$ and $\mathrm{A}$ units were different. Their optimized configuration torsional angles between the $\mathrm{D}$ and $\mathrm{A}$ units of $\sim 44^{\circ}, \sim 61^{\circ}$ and $\sim 44^{\circ} / \sim 61^{\circ}$ were observed for 2TPA-QBT, 2MeTPA-QBT and TPA-QBT-MeTPA, respectively. Density functional theory (DFT) analysis revealed that the emission of TPA-QBT-MeTPA had a more obvious HLCT feature. The doped OLEDs based on TPA-QBT-MeTPA achieved a maximum EQE of 3.02\% with an emission peak at $707 \mathrm{~nm}$ and luminance of $1875 \mathrm{~cd} \mathrm{~m}^{-2}$.

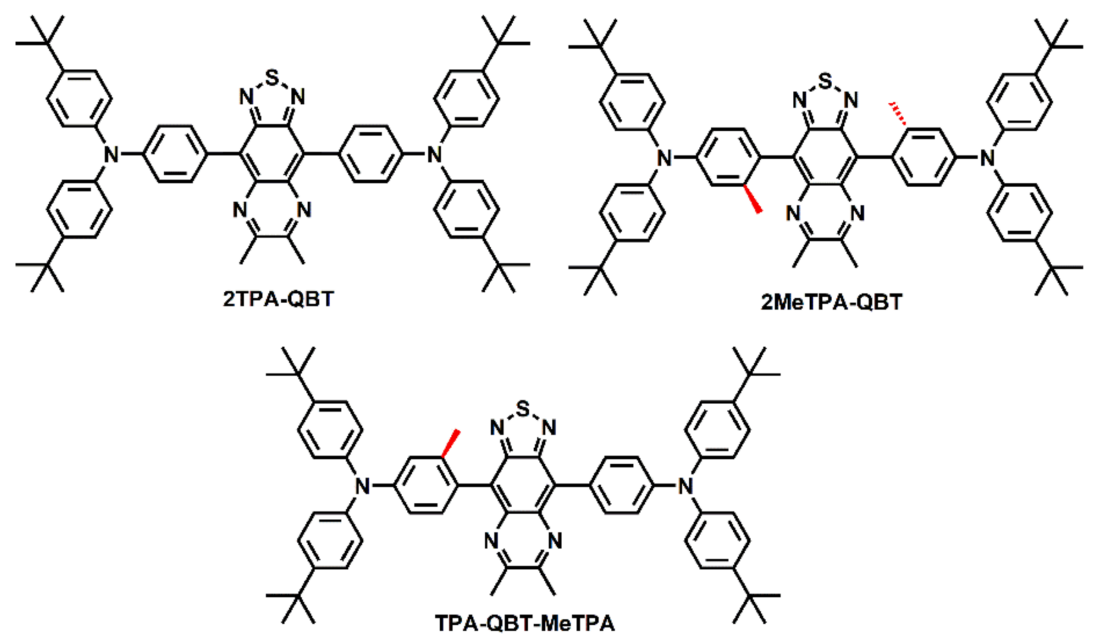

Figure 1. Chemical structures of emitters. 


\section{Results and Discussion}

\subsection{Synthesis and Thermal Stability}

The starting materials and solvents are purchased available and used as received. All operations involving oxygen-sensitive reagents were performed under argon. MeTPABr, TPA-Bpin and MeTPA-Bpin were synthesized according the reported reference by Pd-catalyzed C-N cross-coupling reactions [31]. QBT-2Br was synthesized by aminealdehyde condensation [32]. The final products of 2TPA-QBT, 2MeTPA-QBT and TPAQBT-MeTPA were prepared using the Suzuki coupling reaction using $\mathrm{Pd}\left(\mathrm{PPh}_{3}\right)_{4}$ as a catalyst. All the new compounds were characterized using NMR spectroscopy and mass spectrometry, confirming their well-defined chemical structures. Detailed synthesis processes are provided in the supporting information (Supplementary Material, Figure S1). Figure 2 shows the thermogravimetric analysis curves of obtained emitters under a $\mathrm{N}_{2}$ atmosphere. The decomposition temperatures $\left(T_{d}\right)$ with $5 \%$ weight loss were found to be 473 , 453 and $453{ }^{\circ} \mathrm{C}$ for 2TPA-QBT, 2MeTPA-QBT and TPA-QBT-MeTPA, respectively, which revealed that these organic emitters had enough high thermal stability for the fabrication of OLEDs.



Figure 2. Thermogravimetric analysis curves for 2TPA-QBT, 2MeTPA-QBT and TPA-QBT-MeTPA.

\subsection{Electrochemical Properties}

As depicted in Figure 3, cyclic voltammetry curves for neat films of 2TPA-QBT, 2MeTPAQBT and TPA-QBT-MeTPA coated on a platinum electrode were measured. The calculating formula of $E_{\mathrm{HOMO}}=\left[-\left(E_{\mathrm{Ox}}-0.44\right)-4.8\right] \mathrm{eV}$ and $E_{\mathrm{LUMO}}=\left[-\left(E_{\mathrm{red}}-0.44\right)-4.8\right] \mathrm{eV}$ were used to estimate the HOMO ( $\left.E_{\mathrm{HOMO}}\right)$ and LUMO energy levels ( $\left.E_{\mathrm{LUMO}}\right)$ by the onset oxidation/reduction potentials $\left(E_{\mathrm{ox}} / E_{\text {red }}\right)$, where $0.44 \mathrm{~V}$ is the potential of ferrocene relative to $\mathrm{Ag} / \mathrm{AgCl}$ and $4.8 \mathrm{eV}$ is the energy level of ferrocene with respect to the vacuum energy level [33]. Three approximate reversible redox couples of $0.81 /-0.73,0.82 /-0.88$ and $0.85 /-0.80 \mathrm{~V}$ versus $\mathrm{Ag} / \mathrm{AgCl}$ were found for 2TPA-QBT, 2MeTPA-QBT and TPAQBT-MeTPA (Table 1), respectively, which were attributed to the first oxidation of the donor units and the first reduction of the acceptor unit [31]. Compared to 2TPA-QBT, the oxidation potentials of the emitters increased slightly and the reduction potentials decreased more obviously for both 2MeTPA-QBT and TPA-QBT-MeTPA. Ultimately, this led to a gradual increase in the electrochemical band gaps $\left(E_{\mathcal{C V}}^{g}\right)$ of $1.54,1.70$ and $1.65 \mathrm{eV}$ for 2TPA-QBT, 2MeTPA-QBT and TPA-QBT-MeTPA, respectively. 


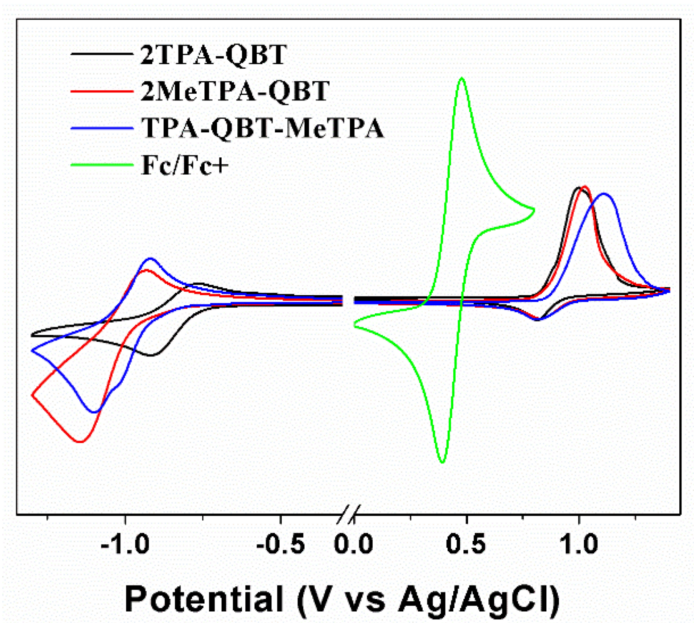

Figure 3. Cyclic voltammograms for emitter films on a platinum electrode in an acetonitrile solution containing $0.1 \mathrm{~mol} \mathrm{~L}^{-1} \mathrm{Bu}_{4} \mathrm{NPF}_{6}$ at a $100 \mathrm{mV} \mathrm{s}^{-1}$ scan rate using a ferrocene internal standard.

Table 1. Thermal stability and electrochemical data for the three emitters.

\begin{tabular}{|c|c|c|c|c|c|c|}
\hline Emitters & $E_{\mathrm{ox}}^{\mathrm{a} / \mathrm{V}}$ & $E_{\text {red }}{ }^{a} / \mathrm{V}$ & $E_{\text {HOMO }} \mathrm{b} / \mathrm{eV}$ & $E_{\text {LUMO }}{ }^{b} / \mathrm{eV}$ & $E_{\mathrm{cv}} / \mathrm{eV}$ & $T_{\mathrm{d}} /{ }^{\circ} \mathrm{C}$ \\
\hline 2TPA-QBT & 0.81 & -0.73 & -5.17 & -3.63 & 1.54 & 473 \\
\hline 2MeTPA-QBT & 0.82 & -0.88 & -5.18 & -3.48 & 1.70 & 453 \\
\hline TPA-QBT-MeTPA & 0.85 & -0.80 & -5.21 & -3.56 & 1.65 & 453 \\
\hline
\end{tabular}

a Onset oxidation and reduction potentials measured by cyclic voltammetry in solid films. ${ }^{\mathrm{b}} E_{\mathrm{HOMO}}=\left[-\left(E_{\mathrm{OX}}\right.\right.$ $-0.44)-4.8] \mathrm{eV}, E_{\mathrm{LUMO}}=\left[-\left(E_{\text {red }}-0.44\right)-4.8\right] \mathrm{eV}$, where $0.44 \mathrm{~V}$ is the value for ferrocene vs. $\mathrm{Ag} / \mathrm{AgCl}$ and $4.8 \mathrm{eV}$ is the energy level of ferrocene relative to the vacuum energy level.

\subsection{Photophysical Properties}

Figure 4 shows the UV-vis absorption and fluorescence spectra of emitters in toluene. The intense absorptions at $<350 \mathrm{~nm}$ were assigned to the intramolecular $\pi-\pi^{*}$ electronic transition. The broad absorptions at 500-700 nm were attributed to intramolecular charge transfer (ICT) transitions from D to A units. Almost the same $\pi-\pi^{*}$ electronic absorptions occurred at $\sim 310 \mathrm{~nm}$ for all three emitters, but different ICT absorption bands were observed (589, 557 and $574 \mathrm{~nm}$ for 2TPA-QBT, 2MeTPA-QBT and TPA-BT-MeTPA, respectively). Due to the increase of torsion in molecular space, the intensity of the ICT absorption bands gradually decreased in the order of 2TPA-QBT $>$ TPA-QBT-MeTPA $>$ 2MeTPA-QBT. This suggested that the intramolecular interactions between $\mathrm{D}$ and $\mathrm{A}$ units correspondingly decreased which were induced by the introduced methyl groups. Moreover, these emitters exhibited similar absorption curves and the absorption peak only changed minimally with the solvent polarities changed, implying a rather small dipolar change in the ground state for different solvents (Supplementary Material, Figures S2-S4) [34-36]. Interestingly, an obvious narrow absorption peak at $368 \mathrm{~nm}$ was observed for 2MeTPA-QBT and TPABT-MeTPA. This may be induced by the hindered rotation between D and A units due to the introduction of methyl groups. Red-shifted absorption spectra were obtained in the neat films due to their stronger intermolecular interaction compared to solution state (Supplementary Material, Figure S5). Based on the onsets of film absorption, we calculated the optical band gaps $\left(E_{g}^{o p t}\right)$ as 1.62, 1.72 and $1.68 \mathrm{eV}$ for 2TPA-QBT, 2MeTPA-QBT and TPA-QBT-MeTPA, respectively. 


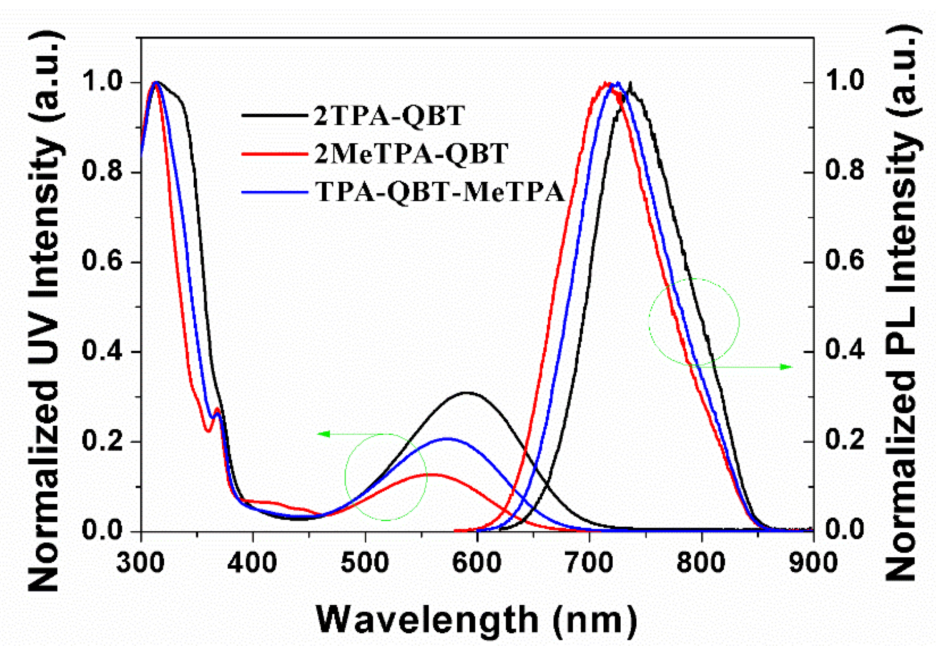

Figure 4. Ultraviolet-visible absorption and photoluminescence spectra for 2TPA-QBT, 2MeTPAQBT and TPA-QBT-MeTPA in toluene solution.

The photoluminescence (PL) spectra in different solvents for emitters are depicted in Supplementary Material, Figures S6-S8. Different from the absorption properties, broadened PL spectra with a remarkable redshift were observed as the solvent polarity increased. The emission peaks shifted from 714, 662 and $694 \mathrm{~nm}$ in nonpolar hexane to 780, 796 and $788 \mathrm{~nm}$ in polar dichloromethane for 2TPA-QBT, 2MeTPA-QBT and TPAQBT-MeTPA, respectively. The large solvatochromic shift indicated a strong interaction between $\mathrm{D}$ and $\mathrm{A}$ unit. Furthermore, the neat film of emitters produced emission peaks at 780, 732 and $748 \mathrm{~nm}$ for 2TPA-QBT, 2MeTPA-QBT and TPA-QBT-MeTPA, respectively (Figures S9-S11). Correspondingly, the PLQYs of the emitters in toluene were 26\%, 38\% and $34 \%$, and in neat films were $5 \%, 6 \%$ and $8 \%$ for 2TPA-QBT, 2MeTPA-QBT and TPAQBT-MeTPA, respectively. However, the PLQYs increased significantly when blended in 4,4'-bis(N-carbazolyl)-1,1-biphenyl (CBP) films of 24\%, 27\% and 31\% compared to in neat films for 2TPA-QBT, 2MeTPA-QBT and TPA-QBT-MeTPA, respectively. The lowered PLQYs in the neat films indicated that these emitters suffered from aggregation-caused quenching (ACQ) due to molecular stacking. Only nanosecond level emission lifetime in the neat film was observed, and the exciton lifetime hardly changes at different temperatures. This is quite different from the TADF and phosphorescence materials, which suggesting that there was no RISC process from the lowest triplet state $\left(\mathrm{T}_{1}\right)$ to the lowest singlet state $\left(\mathrm{S}_{1}\right)$ (Supplementary Material, Figures S12-S14). The resulting photophysical data are summarized in Table 2.

Table 2. Photophysical properties for the three emitters.

\begin{tabular}{cccccccc}
\hline Emitters & $\lambda_{\text {abs sol }} / \mathbf{n m}^{\mathbf{a}}$ & $\lambda_{\text {abs film/nm }}{ }^{\mathbf{b}}$ & $\lambda_{\text {em sol }} / \mathbf{n m}^{\mathbf{a}}$ & $\lambda_{\text {em film }} / \mathbf{n m}^{\mathbf{b}}$ & $E_{g}^{\text {opt }} / \mathbf{e V}^{\mathbf{c}}$ & $\boldsymbol{\Phi}_{\mathrm{F}} / \%_{\text {sol }}{ }^{\mathbf{d}}$ & $\tau_{\text {sol }} / \mathbf{n s}$ \\
\hline 2TPA-QBT & 589,314 & 609 & 736 & 780 & 1.62 & 26 & $6^{\mathrm{a}} / 3^{\mathrm{b}}$ \\
2MeTPA-QBT & 557,312 & 574 & 714 & 732 & 1.72 & 38 & $5^{\mathrm{a} / 6^{\mathrm{b}}}$ \\
TPA-QBT-MeTPA & 557,312 & 585 & 724 & 748 & 1.68 & 34 & $7^{\mathrm{a}} / 3^{\mathrm{b}}$ \\
\hline
\end{tabular}

${ }^{a}$ Measured in toluene $\left(10^{-5} \mathrm{~mol} \mathrm{~L}{ }^{-1}\right) ;{ }^{b}$ measured in neat film; ${ }^{c}$ optical band gaps were determined using $E_{g}^{\text {opt }}$ film $=1240 / \lambda_{\text {onset, film }}$;

$\mathrm{d}$ absolute PL quantum yield measured using an integrating sphere.

Lippert-Mataga expression was used for better understanding the relationship between the Stokes shift and solvent polarity parameter $(f)$ to evaluate the ICT effect [37-39]. As depicted in Figure 5, two-stage linear relations were simulated in respective low and high polarity solvents for all emitters. The dipole moments $\left(\mu_{\mathrm{e}}\right)$ of $12.6,13.8$ and $11.6 \mathrm{D}$ in low polarity solvents, as well as $29.8,31.5$ and $30.7 \mathrm{D}$ in high polarity solvents, were calculated for 2TPA-QBT, 2MeTPA-QBT and TPA-QBT-MeTPA, respectively. Similar to earlier studies, the smaller $\mu_{\mathrm{e}}$ in less polar solvents was attributed to the LE state, while the 
larger $\mu_{\mathrm{e}}$ in highly polar solvents was assigned to the CT excited state. The HLCT state was formed through the coupling and intercrossing between the CT and LE states. More detailed photophysical data are summarized in Table S1 (Supplementary Material).

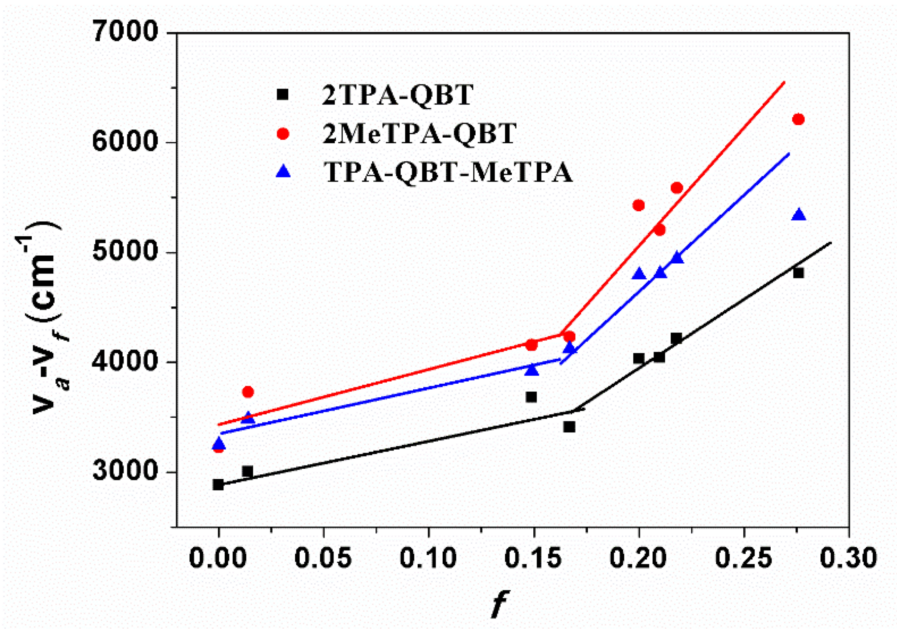

Figure 5. Linear correlation of orientation polarity $f$ of solvent media with the Stokes shift $\left(\mathrm{v}_{a}-\mathrm{v}_{f}\right)$ for all emitters.

\subsection{Theoretical Calculations}

Quantum calculations were performed using the Gaussian 09 suite of programs at the B3LYP/6-31G(d) level for further understanding their photophysical properties [40]. Due to steric hindrance of methyl, different torsional angles of $\sim 44^{\circ}$ for 2TPA-QBT, $\sim 61^{\circ}$ for 2MeTPA-QBT and $\sim 44^{\circ} / \sim 61^{\circ}$ for TPA-QBT-MeTPA between the D and A units were observed in their optimized configuration, respectively (Figure 6a). As expected, the HOMO distributions were mainly located on the D units and the LUMO levels were mostly distributed on the A unit. Furthermore, with the torsion increased, the LUMO were gradually centralized toward the central A unit, while the HOMO were more dispersed on the $\mathrm{D}$ units. Thus, the introduced methyl effectively regulate the overlap of HOMO and LUMO orbitals.

The natural transition orbitals (NTOs) were also further calculated to analyze the electron transition characters (Supplementary Material, Figures S15-S17). For the singlet states $\left(\mathrm{S}_{1}\right)$ of 2 TPA-QBT, the holes were located on the whole molecule and the particles were centralized on the central A component, suggesting the existence of both CT and LE states. In contrast, for the singlet states $\left(S_{1}\right.$ and $\left.S_{2}\right)$ of 2MeTPA-QBT and TPA-QBT-MeTPA, the holes and particles were centralized on the D and A components, respectively, implying that there was only the CT state. For the first-excited triplet state $\left(\mathrm{T}_{1}\right)$ of all emitters, the overlap of the distribution for holes and particles demonstrated the coexistence of CT and LE components. However, for the second-excited triplet state $\left(\mathrm{T}_{2}\right)$, only the CT state was observed for all emitters.

There were large band gaps of $1.23,1.07$ and $1.16 \mathrm{eV}$ between $\mathrm{T}_{2}$ and $\mathrm{T}_{1}$, as well as band gaps of 0.94, 0.95 and $0.95 \mathrm{eV}$ between $S_{1}$ and $T_{1}$ for 2TPA-QBT, 2MeTPA-QBT and TPA-QBT-MeTPA, respectively. These large band gaps may suppress the internal conversion rate from $T_{2}$ to $T_{1}$ and the RISC rate of $T_{1}-S_{1}$ according to the band-gap law. However, small band gaps between $S_{1}$ (and/or $S_{2}$ ) and $T_{2}\left(\right.$ or $T_{3}$ ) were obtained for all emitters, which could facilitate the RISC process from $T_{2}$ (and/or $T_{3}$ ) to $S_{1}$ (and/or $S_{2}$ ). These characteristics of NTOs and excited energy levels were in accordance with the "hot" exciton principle, thereby leading to high production efficiency of radiative singlet excitons $\left(\eta_{\mathrm{S}}\right)$ for these emitters. The computed spin-orbit coupling (SOC) matrix element values of $\xi$ $\left(\mathrm{S}_{1}, \mathrm{~T}_{2}\right) / \xi\left(\mathrm{S}_{1}, \mathrm{~T}_{3}\right)$ were $0.19 / 0.06,0.26 / 0.26$ and $0.32 / 0.36 \mathrm{~cm}^{-1}$ for 2TPA-QBT, 2MeTPAQBT and TPA-QBT-MeTPA, respectively. The larger value of SOC may lead to a more enhanced RISC efficiency for TPA-QBT-MeTPA. 
a)
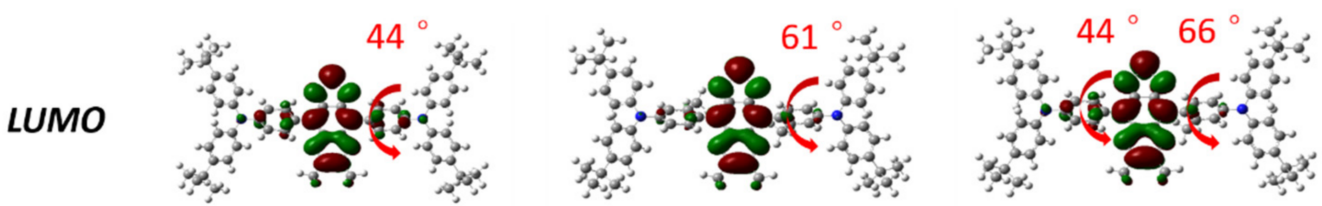

$\frac{-2.72 \mathrm{eV}}{\Delta E=1.88 \mathrm{eV}}$

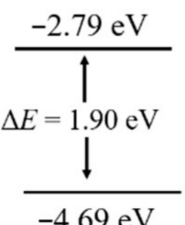

$\frac{-2.74 \mathrm{eV}}{\uparrow}$

$\Delta E=1.89 \mathrm{eV}$

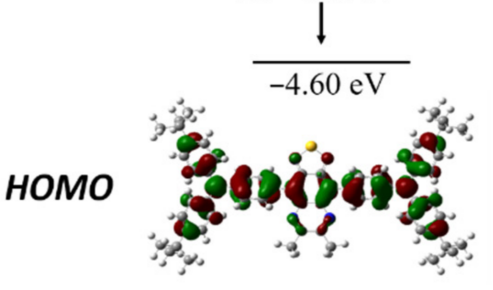

2TPA-QBT
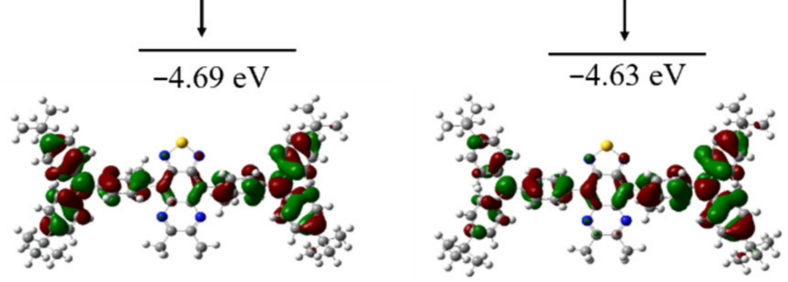

2MeTPA-QBT

TPA-QBT-MeTPA
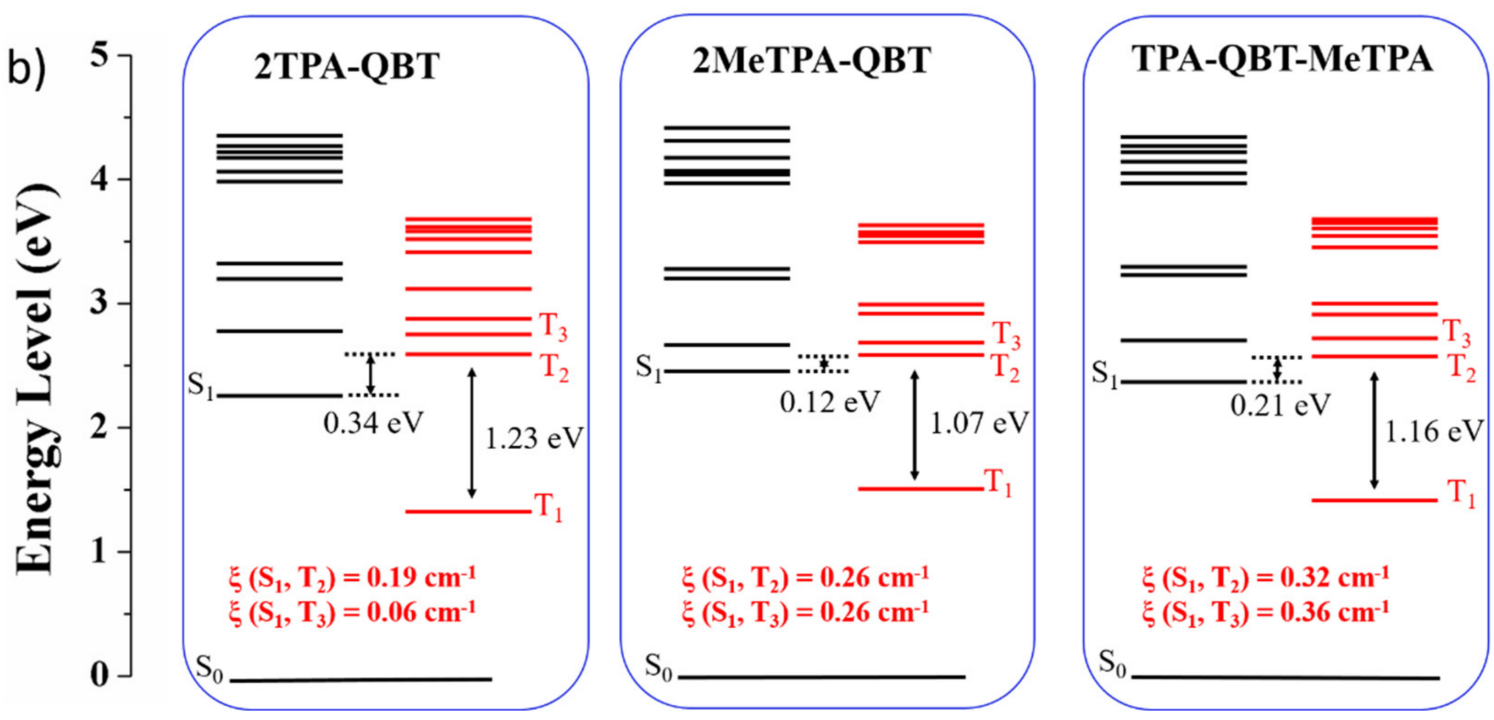

Figure 6. (a) Optimized structure and calculated HOMO/LUMO spatial distributions and (b) energy level diagrams of emitters.

\subsection{Electroluminescence Properties}

To research their electroluminescence (EL) properties, OLEDs were fabricated by using these emitters as dopants with the configuration of indium tin oxide (ITO)/1,1-bis[4[N,N'-di(p-tolyl)amino]-phenyl]-cyclohexane (TAPC, $30 \mathrm{~nm}) / 4,4^{\prime}, 4^{\prime \prime}$-tris(carbaz;tris $(4-(9 \mathrm{H}-$ carbazol-9-yl) (TCTA, $15 \mathrm{~nm}$ )/emitter: 4,4'-bis( $N$-carbazolyl)-1,1'-biphenyl (CBP, $5 \mathrm{wt} . \%$, $15 \mathrm{~nm}$ )/1,3,5-tri[(3-pyridyl)phen-3-yl]benzene (TmPyPB, $65 \mathrm{~nm}) / \mathrm{LiF}(1 \mathrm{~nm}) / \mathrm{Al}(100 \mathrm{~nm})$, where CBP, TAPC, TCTA and TmPyPB served as the host material, hole injection, hole transporting and electron transporting layers, respectively (Figure 7). Emitters were doped into the CBP host with an optimal doping concentration of $5 \mathrm{wt} . \%$ to serve as the EMLs. 


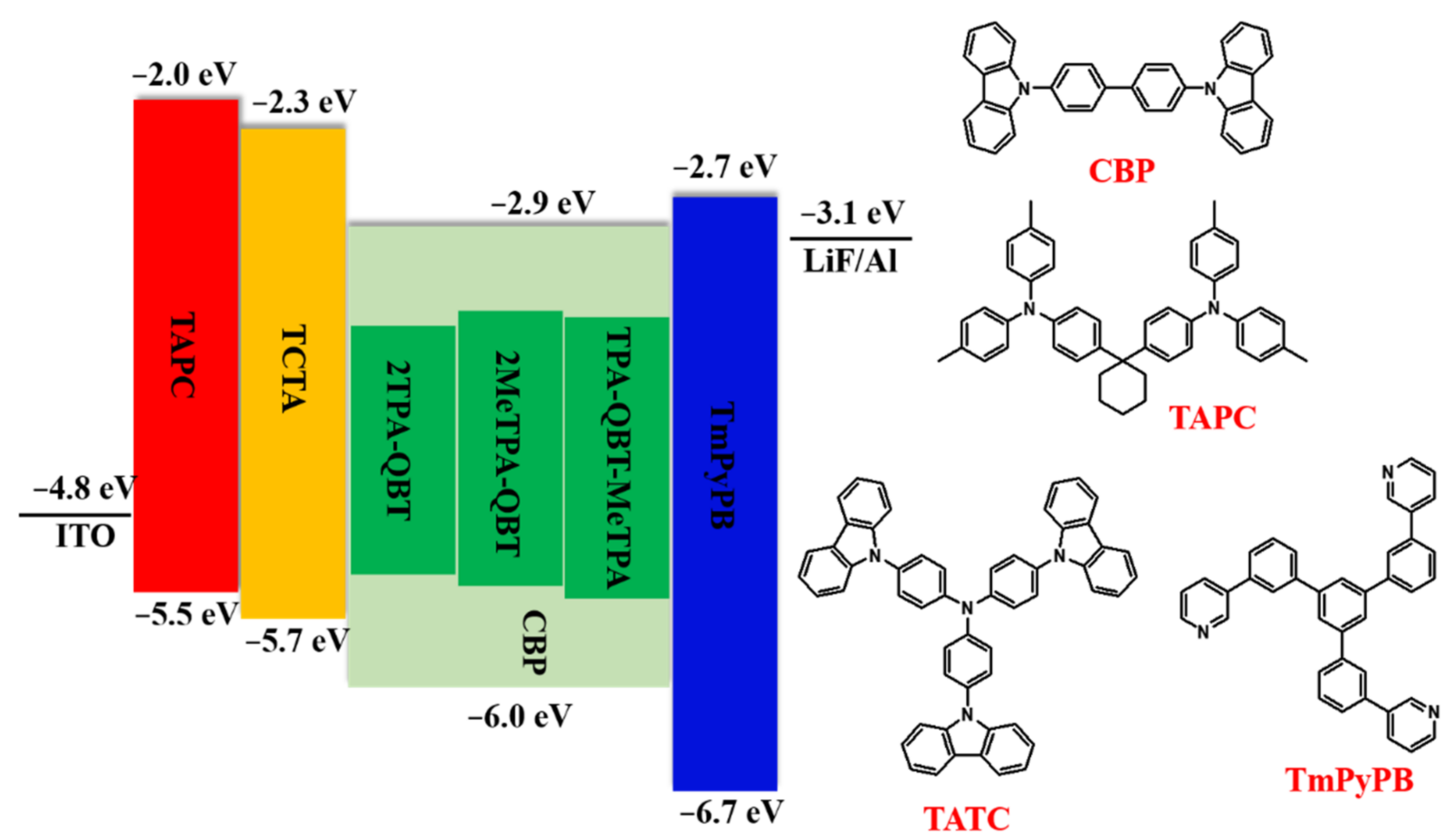

Figure 7. Energy diagram of OLEDs and the chemical structures of adopted materials.

As shown in Figure 8, the devices exhibited emission peaks at 718, 693 and $707 \mathrm{~nm}$ for the 2TPA-QBT-, 2MeTPA-QBT- and TPA-QBT-MeTPA-based OLEDs, respectively. Compared to 2TPA-QBT, the EL peaks of the 2MeTPA-QBT- and TPA-QBT-MeTPA-based OLEDs showed obvious blue shifts of 25 and $11 \mathrm{~nm}$ from the PL maxima for the corresponding neat films. The devices exhibited maximum external quantum efficiencies (EQE $E_{\max }$ ) of $1.58 \%, 1.33 \%$ and 3.02\% for the 2TPA-QBT-, 2MeTPA-QBT- and TPA-QBTMeTPA-doped OLEDs with Commission Internationalede L'Eclairage (CIE) coordinates of $(0.70,0.31),(0.70,0.29)$ and $(0.69,0.30)$, turn-on voltages $\left(V_{\text {on }}\right)$ of $5.6,8.3$ and $4.4 \mathrm{~V}$ and maximum luminance values $\left(L_{\max }\right)$ of 783,839 and $1875 \mathrm{~cd} \mathrm{~m}^{-2}$ for 2TPA-QBT-, 2MeTPAQBT- and TPA-QBT-MeTPA-based OLEDs, respectively. Furthermore, the PLQYs of the active layer composed of emitter:CBP (5 wt. \%) were measured, with values of $24 \%, 27 \%$ and 31\% obtained for the 2TPA-QBT-, 2MeTPA-QBT- and TPA-QBT-MeTPA-doped films, respectively. The EL data for all the emitters are summarized in Table 3.

a)

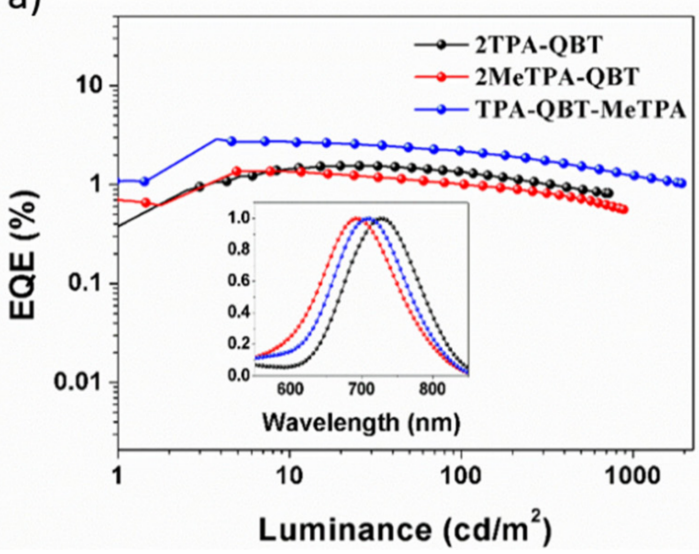

b)

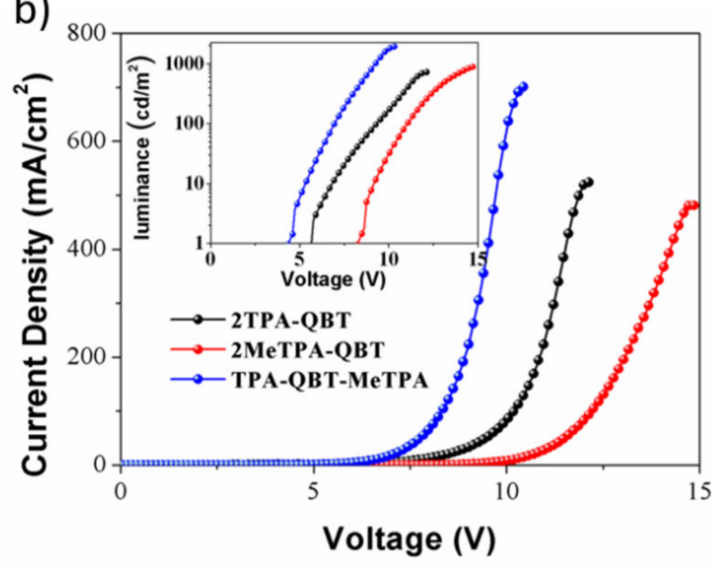

Figure 8. (a) External quantum efficiency-luminance curves. Inset: normalized EL spectra of the devices. (b) Current density-voltage-luminance curves. 
Table 3. Electroluminescence parameters for the three doped devices.

\begin{tabular}{cccccc}
\hline Emitters & $\lambda_{\text {EL }}(\mathbf{n m})^{\mathbf{a}}$ & $\mathbf{V}_{\text {on }}(\mathbf{V})^{\mathbf{b}}$ & ${\text { EQE }(\%)^{\mathbf{c}}}^{\mathbf{c}}$ & $L_{\max }\left(\mathbf{c d} / \mathbf{m}^{\mathbf{2}}\right)^{\mathbf{d}}$ & CIE $^{\mathbf{e}}$ \\
\hline 2TPA-QBT & 718 & 5.6 & 1.58 & 783 & $(0.70,0.31)$ \\
2MeTPA-QBT & 693 & 8.3 & 1.33 & 839 & $(0.70,0.29)$ \\
TPA-QBT-MeTPA & 707 & 4.4 & 3.02 & 1875 & $(0.69,0.30)$ \\
\hline
\end{tabular}

${ }^{a}$ Maximum EL emission peak; ${ }^{b}$ turn-on voltage; ${ }^{c}$ maximum external quantum efficiency; ${ }^{d}$ maximum luminance

e color coordinates.

\section{Conclusions}

To summarize, three D-A-D-type NIR emitters (2TPA-QBT, 2MeTPA-QBT and TPAQBT-MeTPA) were designed and synthesized. Introducing methyl steric hindrance between the D and A units can cause different torsional angles, and further regulate the overlap of HOMO and LUMO orbitals. The emission of the emitters proved to be governed by an HLCT mechanism. These emitters showed different emission wavelengths with acceptable PLQYs in the toluene and neat film. OLEDs using these emitters as single dopant exhibited NIR emission with EL peaks at 718, 693 and $707 \mathrm{~nm}$ and maximum EQEs of $1.58 \%, 1.33 \%$ and $3.02 \%$ for 2TPA-QBT-, 2MeTPA-QBT- and TPA-QBT-MeTPA-doped OLEDs, respectively. This work showed the effect of spatial configuration changes on the luminescence properties of NIR organic emitters.

Supplementary Materials: The supplementary materials are available online. Figure S1. Synthetic routes of 2TPA-QBT, 2MeTPA-QBT and TPA-QBT-MeTPA. Figure S2. UV-vis absorption spectra of 2TPA-QBT in different solvents. Figure S3. UV-vis absorption spectra of 2MeTPAQBT in different solvents. Figure S4. UV-vis absorption spectra of TPA-QBT-MeTPA in different solvents. Figure S5. UV-vis absorption spectra of emitters in neat films. Figure S6. Photoluminescence spectra of 2TPA-QBT in different solvents. Figure S7. Photoluminescence spectra of 2MeTPA-QBT in different solvents. Figure S8. Photoluminescence spectra of TPA-QBT-MeTPA in different solvents. Figure S9. Variable-temperaturephotoluminescence spectra of 2TPA-QBT in neat films. Figure S10. Variable-temperaturephotoluminescence spectra of 2MeTPA-QBT in neat films. Figure S11. Variable-temperaturephotoluminescence spectra of TPA-QBT-MeTPA in neat films. Figure S12. Variable-temperaturephotoluminescence decay characteristics of TPA-QBT-MeTPA in neat films. Figure S13. Variable-temperaturephotoluminescence decay characteristics of 2MeTPAQBT in neat films. Figure S14. Variable-temperaturephotoluminescence decay characteristics of TPA-QBT-MeTPA in neatfilms. Figure S15. The energy level and the natural transitionorbitals of 2TPA-QBT. Figure S16. The energy level and the natural transitionorbitals of 2MeTPA-QBT. Figure S17. The energy level and the natural transitionorbitals of TPA-QBTMeTPA. Table S1. Photophysical properties for the three emitters.

Author Contributions: Y.Z., C.W., M.Z., J.M. contributed to methodology and writing of the paper. All authors have read and agreed to the published version of the manuscript.

Funding: This research received no external funding.

Institutional Review Board Statement: Not applicable.

Informed Consent Statement: Not applicable.

Data Availability Statement: Data on the compounds are available from the authors.

Acknowledgments: This research was financially supported by the National Natural Science Foundation of China (51903157 and 51903158), the Shenzhen Science and Technology Program (KQTD20170330110107046 and JCYJ20190808151209557) and the China Postdoctoral Science Foundation Funded Project (2017M622748, 2019T120747). We thank the Instrumental Analysis Center of Shenzhen University for analytical support.

Conflicts of Interest: The authors declare no conflict of interest.

Sample Availability: Samples of the compounds are available from the authors. 


\section{References}

1. Wang, X.Q.; Hu, Y.; Yu, Y.J.; Tian, Q.S.; Shen, W.S.; Yang, W.Y.; Jiang, Z.Q.; Liao, L.S. Over 800 nm Emission via Harvesting of Triplet Excitons in Exciplex Organic Light-Emitting Diodes. J. Phys. Chem. Lett. 2021, 12, 6034-6040. [CrossRef]

2. Babu Kajjam, A.; Vaidyanathan, S. Acenaphthene-imidazole based red-to-NIR Emissive Homoleptic and Heteroleptic Ir(III) complexes for OLEDs: Combined experimental and theoretical approach. Inor. Chim. Acta 2021, 519, 120268. [CrossRef]

3. Yang, K.; Wang, L.; Zhang, Q. Preparation and properties of a flexible night vision imaging system filter for avionic LED displays. J. Mater. Sci.-Mater. Electron. 2015, 26, 2222-2229. [CrossRef]

4. Strobl, M.; Mayr, T.; Klimant, I.; Borisov, S.M. Photostable upconverting and downconverting pH sensors based on combination of a colorimetric NIR indicator and stable inorganic phosphors as secondary emitters. Sens. Actuators B-Chem. 2017, 245, 972-979. [CrossRef]

5. Xiang, H.; Cheng, J.; Ma, X.; Zhou, X.; Chruma, J.J. Near-infrared phosphorescence: Materials and applications. Chem. Soc. Rev. 2013, 42, 6128-6185. [CrossRef]

6. $\quad$ Qian, G.; Zhong, Z.; Luo, M.; Yu, D.; Zhang, Z.; Wang, Z.Y.; Ma, D. Simple and Efficient Near-Infrared Organic Chromophores for Light-Emitting Diodes with Single Electroluminescent Emission above $1000 \mathrm{~nm}$. Adv. Mater. 2009, 21, 111-116. [CrossRef]

7. Wang, C.; Li, X.-L.; Gao, Y.; Wang, L.; Zhang, S.; Zhao, L.; Lu, P.; Yang, B.; Su, S.-J.; Ma, Y. Efficient Near-Infrared (NIR) Organic Light-Emitting Diodes Based on Donor-Acceptor Architecture: An Improved Emissive State from Mixing to Hybridization. Chem. Mater. 2008, 20, 6208-6216. [CrossRef]

8. Xiong, W.; Meng, F.; Tan, H.; Wang, Y.; Wang, P.; Zhang, Y.; Tao, Q.; Su, S.; Zhu, W. Dinuclear platinum complexes containing aryl-isoquinoline and oxadiazole-thiol with an efficiency of over $8.8 \%$ : In-depth investigation of the relationship between their molecular structure and near-infrared electroluminescent properties in PLEDs. J. Mater. Chem. C 2016, 4, 6007-6015. [CrossRef]

9. Leppenen, N.V.; Lanco, L.; Smirnov, D.S. Quantum Zeno effect and quantum nondemolition spin measurement in a quantum dot-micropillar cavity in the strong coupling regime. Phys. Rev. B 2021, 103, 045413. [CrossRef]

10. Yao, L.; Zhang, S.; Wang, R.; Li, W.; Shen, F.; Yang, B.; Ma, Y. Highly efficient near-infrared organic light-emitting diode based on a butterfly-shaped donor-acceptor chromophore with strong solid-state fluorescence and a large proportion of radiative excitons. Angew. Chem. Int. Ed. 2014, 53, 2119-2123. [CrossRef] [PubMed]

11. Zhu, X.H.; Peng, J.; Cao, Y.; Roncali, J. Solution-processable single-material molecular emitters for organic light-emitting devices. Chem. Soc. Rev. 2011, 40, 3509-3524. [CrossRef] [PubMed]

12. You, C.; Liu, D.; Yu, J.; Tan, H.; Zhu, M.; Zhang, B.; Liu, Y.; Wang, Y.; Zhu, W. Boosting Efficiency of Near-Infrared Emitting Iridium(III) Phosphors by Administrating Their $\pi-\pi$ Conjugation Effect of Core-Shell Structure in Solution-Processed OLEDs. Adv. Opt. Mater. 2020, 8, 2000154. [CrossRef]

13. Chaaban, M.; Chi, Y.C.; Worku, M.; Zhou, C.; Lin, H.; Lee, S.; Ben-Akacha, A.; Lin, X.; Huang, C.; Ma, B. Thiazol-2-thiolate-Bridged Binuclear Platinum(II) Complexes with High Photoluminescence Quantum Efficiencies of up to Near Unity. Inorg. Chem. 2020, 59, 13109-13116. [CrossRef]

14. Kajjam, A.B.; Vaidyanathan, S. Structural Mimics of Phenyl Pyridine (ppy)—Substituted, Phosphorescent Cyclometalated Homo and Heteroleptic Iridium(III) Complexes for Organic Light Emitting Diodes-An Overview. Chem. Rec. 2018, 18, 293-349. [CrossRef]

15. Chaskar, A.; Chen, H.F.; Wong, K.T. Bipolar host materials: A chemical approach for highly efficient electrophosphorescent devices. Adv. Mater. 2011, 23, 3876-3895. [CrossRef]

16. Cebrian, C.; Mauro, M. Recent advances in phosphorescent platinum complexes for organic light-emitting diodes. Beilstein. J. Org. Chem. 2018, 14, 1459-1481. [CrossRef]

17. Ganesan, P.; Hung, W.Y.; Tso, J.Y.; Ko, C.L.; Wang, T.H.; Chen, P.T.; Hsu, H.F.; Liu, S.H.; Lee, G.H.; Chou, P.T.; et al. Functional Pyrimidinyl Pyrazolate Pt(II) Complexes: Role of Nitrogen Atom in Tuning the Solid-State Stacking and Photophysics. Adv. Funct. Mater. 2019, 29, 1900923. [CrossRef]

18. Lee, J.; Jeong, C.; Batagoda, T.; Coburn, C.; Thompson, M.E.; Forrest, S.R. Hot excited state management for long-lived blue phosphorescent organic light-emitting diodes. Nat. Commun. 2017, 8, 15566. [CrossRef]

19. Tuong Ly, K.; Chen-Cheng, R.-W.; Lin, H.-W.; Shiau, Y.-J.; Liu, S.-H.; Chou, P.-T.; Tsao, C.-S.; Huang, Y.-C.; Chi, Y. Near-infrared organic light-emitting diodes with very high external quantum efficiency and radiance. Nat. Photonics 2016, 11, 63-68. [CrossRef]

20. Tang, X.; Lee, Y.-T.; Feng, Z.; Ko, S.Y.; Wu, J.W.; Placide, V.; Ribierre, J.-C.; D'Aléo, A.; Adachi, C. Color-Tunable Low-Threshold Amplified Spontaneous Emission from Yellow to Near-Infrared (NIR) Based on Donor-Spacer-Acceptor-Spacer-Donor Linear Dyes. Angew. Chem. Int. Ed. 2016, 55, 5739-5744. [CrossRef]

21. Yang, Z.; Mao, Z.; Xie, Z.; Zhang, Y.; Liu, S.; Zhao, J.; Xu, J.; Chi, Z.; Aldred, M.P. Recent advances in organic thermally activated delayed fluorescence materials. Chem. Soc. Rev. 2017, 46, 915-1016. [CrossRef] [PubMed]

22. Yuan, Y.; Hu, Y.; Zhang, Y.-X.; Lin, J.-D.; Wang, Y.-K.; Jiang, Z.-Q.; Liao, L.-S.; Lee, S.-T. Over 10\% EQE Near-Infrared Electroluminescence Based on a Thermally Activated Delayed Fluorescence Emitter. Adv. Funct. Mater. 2017, 27, 1700986. [CrossRef]

23. Tang, X.; Li, X.-L.; Liu, H.; Gao, Y.; Shen, Y.; Zhang, S.; Lu, P.; Yang, B.; Su, S.-J.; Ma, Y. Efficient near-infrared emission based on donor-acceptor molecular architecture: The role of ancillary acceptor of cyanophenyl. Dye Pigm. 2018, 149, 430-436. [CrossRef]

24. Ganesan, P.; Chen, D.-G.; Chen, W.-C.; Gnanasekaran, P.; Lin, J.-A.; Huang, C.-Y.; Chen, M.-C.; Lee, C.-S.; Chou, P.-T.; Chi, Y. Methoxy substituents activated carbazole-based boron dimesityl TADF emitters. J. Mater. Chem. C 2020, 8, 4780-4788. [CrossRef] 
25. Chen, L.; Zhang, S.; Li, H.; Chen, R.; Jin, L.; Yuan, K.; Li, H.; Lu, P.; Yang, B.; Huang, W. Breaking the Efficiency Limit of Fluorescent OLEDs by Hybridized Local and Charge-Transfer Host Materials. J. Phys. Chem. Lett. 2018, 9, 5240-5245. [CrossRef] [PubMed]

26. Chen, X.; Yang, Z.; Li, W.; Mao, Z.; Zhao, J.; Zhang, Y.; Wu, Y.C.; Jiao, S.; Liu, Y.; Chi, Z. Nondoped Red Fluorophores with Hybridized Local and Charge-Transfer State for High-Performance Fluorescent White Organic Light-Emitting Diodes. ACS Appl. Mater. Interfaces 2019, 11, 39026-39034. [CrossRef] [PubMed]

27. Jiang, Y.; Hu, Z.; Zhou, B.; Zhong, C.; Sun, Z.; Sun, H. Accurate Prediction for Dynamic Hybrid Local and Charge Transfer Excited States from Optimally Tuned Range-Separated Density Functionals. J. Phys. Chem. C 2019, 123, 5616-5625. [CrossRef]

28. Song, J.; Lee, H.; Jeong, E.G.; Choi, K.C.; Yoo, S. Organic Light-Emitting Diodes: Pushing Toward the Limits and Beyond. Adv. Mater. 2020, 32, 1907539. [CrossRef]

29. Liu, T.; Xie, G.; Zhong, C.; Gong, S.; Yang, C. Boosting the Efficiency of Near-Infrared Fluorescent OLEDs with an Electroluminescent Peak of Nearly $800 \mathrm{~nm}$ by Sensitizer-Based Cascade Energy Transfer. Adv. Funct. Mater. 2018, 28, 1706088. [CrossRef]

30. Li, C.; Duan, R.; Liang, B.; Han, G.; Wang, S.; Ye, K.; Liu, Y.; Yi, Y.; Wang, Y. Deep-Red to Near-Infrared Thermally Activated Delayed Fluorescence in Organic Solid Films and Electroluminescent Devices. Angew. Chem. Int. Ed. 2017, 56, 11525-11529. [CrossRef] [PubMed]

31. Zhang, Y.; Zhou, X.; Zhou, C.; Su, Q.; Chen, S.; Song, J.; Wong, W.-Y. High-efficiency organic electroluminescent materials based on the D-A-D type with sterically hindered methyl groups. J. Mater. Chem. C 2020, 8, 6851-6860. [CrossRef]

32. Qian, G.; Zhong, Z.; Luo, M.; Yu, D.; Zhang, Z.; Ma, D.; Wang, Z.Y. Synthesis and Application of ThiadiazoloquinoxalineContaining Chromophores as Dopants for Efficient Near-Infrared Organic Light-Emitting Diodes. J. Phys. Chem. C 2009, 113, 1589-1595. [CrossRef]

33. Cardona, C.M.; Li, W.; Kaifer, A.E.; Stockdale, D.; Bazan, G.C. Electrochemical considerations for determining absolute frontier orbital energy levels of conjugated polymers for solar cell applications. Adv. Mater. 2011, 23, 2367-2371. [CrossRef] [PubMed]

34. Liu, X.; Yu, Z.; Yu, M.; Zhang, X.; Xu, Y.; Lv, P.; Chu, S.; Liu, C.; Lai, W.Y.; Huang, W. Iridium(III)-Complexed Polydendrimers for Inkjet-Printing OLEDs: The Influence of Solubilizing Steric Hindrance Groups. J. Mater. Chem. C 2019, 7, 1880-1887. [CrossRef] [PubMed]

35. Zhang, Y.; Wu, J.; Song, J.; Chen, Z.; He, J.; Wang, X.; Liu, H.; Chen, S.; Qu, J.; Wong, W.-Y. Achieving High-Performance Solution-Processed Deep-Red/Near-Infrared Organic Light-Emitting Diodes with a Phenanthroline-Based and Wedge-Shaped Fluorophore. Adv. Electron. Mater. 2019, 5, 1800677. [CrossRef]

36. Xiao, S.; Zhang, S.-T.; Gao, Y.; Yang, X.; Liu, H.; Li, W.; Yang, B. Efficient and stable deep-blue narrow-spectrum electroluminescence based on hybridized local and charge-transfer (HLCT) state. Dye Pigm. 2021, 193, 109482. [CrossRef]

37. Lippert, V.E. Spektroskopische Bestimmung des Dipolmomentes aromatischer Verbindungen im ersten angeregten Singulettzustand. Electrochemistry 1957, 61, 962-975.

38. Bozkurt, E.; Gul, H.I.; Mete, E. Solvent and substituent effect on the photophysical properties of pyrazoline derivatives: A spectroscopic study. J. Photochem. Photobiol. A 2018, 352, 35-42. [CrossRef]

39. Kumar Satpati, A.; Kumbhakar, M.; Kumar Maity, D.; Pal, H. Photophysical investigations of the solvent polarity effect on the properties of coumarin-6 dye. Chem. Phys. Lett. 2005, 407, 114-118. [CrossRef]

40. Frisch, M.J.; Trucks, G.W.; Schlegel, H.B.; Scuseria, G.E.; Robb, M.A.; Cheeseman; Montgomery, J.R.J.A.; Vreven, T.; Kudin, K.N.; Burant, J.C.; et al. Gaussian 03, Revisionc.02; Gaussian Inc.: Wallingford, CT, USA, 2004. 